\title{
Study on Brain Plasticity of EEG Signals Based on Motor Imagination Training
}

\author{
Yu Rui, Yin Kuiying \\ Nanjing Research Institute of Electronic Technology, Nanjing, China \\ Email address: \\ yurui1244@163.com (Yu Rui), 29525987@qq.com (Yin Kuiying)
}

\section{To cite this article:}

Yu Rui, Yin Kuiying. Study on Brain Plasticity of EEG Signals Based on Motor Imagination Training. Science Innovation. Vol. 8, No. 2, 2020, pp. 48-53. doi: 10.11648/j.si.20200802.16

Received: March 24, 2020; Accepted: May 6, 2020; Published: May 19, 2020

\begin{abstract}
To explore the effects of hand motion imagery on brain plasticity for use in guiding disabled people to use robotic arms, this paper designed a comparison experiment between the disabled and normal people, and collected the EEG data of 4 subjects ( 2 disabled men who lost their right hands and 2 normal men) for five weeks of training. What's more, this paper compared and analyzed the collected EEG rhythm through the brain topographic map. The results showed that after training, the disabled people could produce ERD in both the $\mu$ and $\beta$ frequency bands, and the longer the training time, the more obvious the ERD phenomenon was. This paper also uses the common space pattern algorithm and support vector machine to extract and classify the features of EEG signals. The results show that the classification accuracy of the disabled can reach more than $85 \%$, and that of the normal can reach more than $90 \%$. Based on the results of brain topographical map and classification, this paper concludes that motor imagination training can have a positive effect on the brain of people with impaired motor area, which provides a neurophysiological basis for the extensive application of motor imagination training in the field of rehabilitation.
\end{abstract}

Keywords: Motion Imagination, Brain Plasticity, Brain Topographic Map, Feature Extraction

\section{基于运动想象训练的脑电信号的大脑可塑性研究}

\section{余瑞, 尹奎英}

南京电子技术研究所, 南京, 中国

邮箱

yurui1244@163.com（余瑞），29525987@qq.com（尹奎英）

摘要: 探索手部运动想象对大脑可塑性的影响以便用于指导残疾人使用机械手臂, 本文设计残疾人和正常人的比较实 验, 采集了 4 名被试 (2名失去右手的残疾男性, 2 名正常的男性) 训练五周的脑电数据, 并通过脑地形图对采集的脑电 信号 (EEG)节律进行对比分析。结果表明, 经过训练, 残疾人在 $\mu$ 和 $\beta$ 频段均能产生ERD现象, 并且一段时间内, 训练 时间越长, ERD现象越明显。本文还利用共同空间模式算法和支持向量机对脑电信号进行特征提取及分类，残疾人分 类正确率可以达到 $85 \%$ 以上，正常人可以达到 $90 \%$ 以上。综合脑地形图和分类结果，本文得出了运动想象训练可以对 大脑运动区受损的人的大脑产生积极影响, 这为运动想象训练在康复领域中的广泛应用提供神经生理依据。

关键词：运动想象，大脑可塑性，脑地形图，特征提取 


\section{1. 引言}

神经的可塑性和大脑功能重组是神经损伤后功能恢 复的基础, 是近 20 年来神经生物学研究发展的重要领域。 神经可塑性描述了人类中枢神经系统的内在性质, 从结 构和功能上解释了人类如何适应变化获得新的技能 [1]。 大脑发育成熟后依然保持着一定的可塑性, 可以通过学 习和训练恢复某些大脑损伤。在我们生活中, 有一些群 体因为各种原因导致手臂残疾或者手部运动功能丧失, 他们从心理或者生理上都受到了一定的影响。手部残疾 也影响了大脑运动区的某些结构, 由于残肢不能运动, 久而久之就会遗忘一些手部动作。本文则基于大脑可塑 性理论基础, 研究残疾人运动想象训练过程中脑电信号 的变化情况, 从脑地形图不同节律信号的能量分布角度 进行分析。

脑-机接口（Brain-Computer Interface, BCI）技术的出 现使人脑与外界环境的直接通讯成为可能 [2]。基于运动想 象的脑-机接口从 BCI 应用的角度看, 具有较高的分类准 确率, 并且能够实现多任务的模式; 从脑功能研究的角度 看, 它能够作为运动控制和运动意图研究的平台。BCI 技 术研究的关键是如何快速有效地提取脑电( EEG)的特征 和提高识别准确率。目前基于BCI脑电信号的特征提取的 算法有很多, 如功率谱分析法, 自适应自回归(AAR)模型 法, 独立分量分析(ICA) [3], 共同空间模式(common spatial pattern,CSP) [4], 小波包分析法[5]等。功率谱分析法可以 反映脑电信号的能量变化, 但是会损失信号的时间信息 [6]。 AAR模型法需要的计算量较小且不需要相关频带的先验 知识, 但是对高度非平稳信号效果不佳[7]。ICA算法对采 集的脑电信号数据进行独立分量提取, 一般很难得到有价 值的结果 $[8]$ 。CSP算法的基本原理是基于两个协方差矩阵 的同时对角化来设计最优的空间滤波器, 以分辨左右手想 象运动的脑电特征, 因此不需要对被试者选择特定的频带 进行特征提取 [9]。

$\mathrm{CSP}$ 是近几年来使用率比较高的算法之一，并且支持 向量机(Support Vector Machine, SVM) 的使用范围比较广, 分类效果好。因此本文使用CSP和SVM的方法对运动功能 区的 25 导联的 EEG 数据进行特征提取并分类, 得到了较 好的分类效果。

\section{2. 实验内容}

\section{1. 实验设备及环境}

本文使用的实验系统装置是博瑞康的无线数字脑电 采集系统, 包括NEUSEN W系列的采集软件、放大器和59 导联电极帽。电极帽的电极按照国际标准 $10 / 20$ 导联系统
设置, 参考电极 $(\mathrm{Ref})$ 位于大脑中央区 $(\mathrm{CPz})$, 接地电极 (GND) 位于前额（AFz）, 位置如图 1所示, 所有电极 的阻抗都调至 $20 \mathrm{k} \Omega$ 以下。该设备可以连接Eprime和 Phytoolbox等心理学分析软件, 本文采用后者设计实验流 程。数据采集过程中, 我们将采集系统的参数做如下设置: 采样率为 $1000 \mathrm{~Hz}$ ，滤波范围为 $2-100 \mathrm{~Hz}$ 。

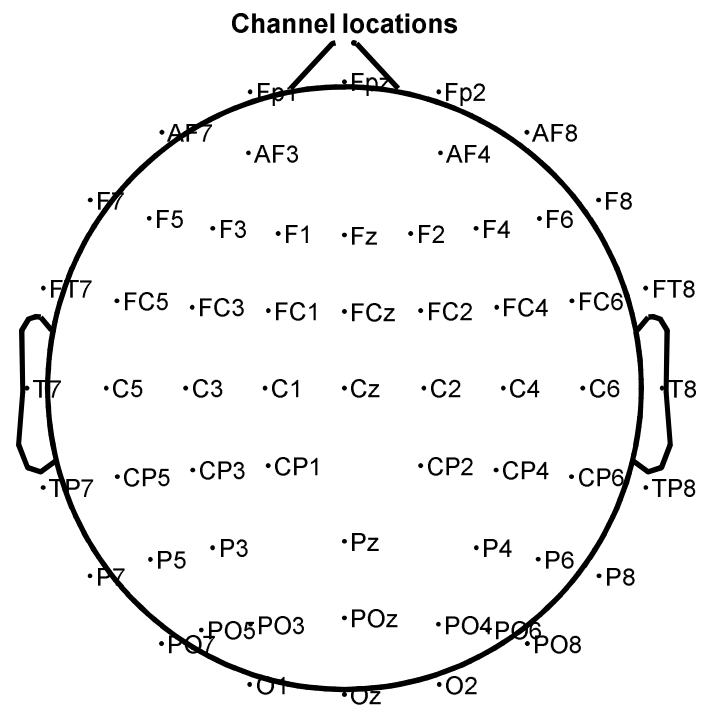

图1 EEG采集电极位置图（Ref和GND未显示）。

\section{2. 实验内容}

实验选取四名受试者（均为男性）, 其中两名为失去 左手的残疾人, 两名为正常人, 所有被试都签署了知情同 意书。实验为期五周, 要求受试者每天进行一个小时的运 动想象训练, 每周末采集一次脑电信号。实验过程中受试 者自然放松地坐在电磁屏蔽的实验室中, 眼睛距离 15 英寸 的电脑显示器 $80 \mathrm{~cm}$, 视角约为 $15^{\circ}$ 。每名受试者按照屏幕 提示完成相应的手部动作想象。

实验范式如图2所示。一次实验由三个阶段完成, 每 两个阶段之间休息两分钟。每当计算机发出 “哔”的一声提 示音, 代表一个实验阶段开始, 受试者进入准备状态。1s 后, 屏幕上出现运动想象类别提示符 （匹代表左手， $\rightarrow$ 代表右手)，受试者随即开始对应的运动想象。提示符持 续显示 $3 \mathrm{~s}$ 后消失, 同时受试者进入休息状态, 持续 $2 \mathrm{~s}$ 。为 了防止提示符出现的规律性使实验受试者产生条件反射 而影响实验结果, 实验范式设计中的两种箭头符号是随机 出现的。每个阶段左手和右手的想象任务次数完全相同, 均为 40 次。一次实验大概需要 24 分钟, 共采集 $120 \times 2$ 个动 作。本实验一共有四名被试, 每人训练五周, 外加初始对 照实验, 我们一共采集了 $120 \times 2 \times 6 \times 4$ 个动作。

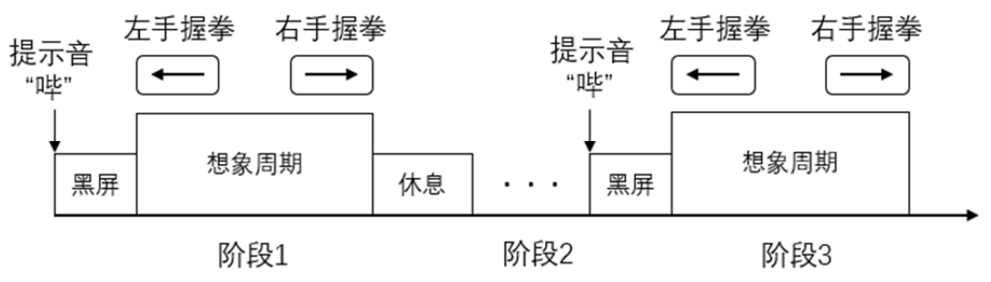

图2 BCI实验范式。 


\section{3. 基本原理}

\section{1. 共同空间模式}

共同空间模式（Common Spatial Pattern, CSP）是由 Muller Gerking 等提出的一种特征提取算法[10]。CSP算 法的目的是寻找一个空间滤波器, 使待分类的两类信号经 过空间滤波后, 最大限度的被区分, 其算法是基于两个协 方差矩阵同时对角化, 使其中一类信号方差最大的同时另 一类信号的方差最小。CSP算法的简单描述如下 $[11,12]$ :

用 $X_{i}, i \in\{1,2\}$ 表示左、右手运动想象模式下的脑电信 号, 那么样本归一化的协方差矩阵为:

$$
R_{i}=\left(X_{i} X_{i}^{T}\right) /\left(\operatorname{trace}\left(X_{i} X_{i}^{T}\right)\right)
$$

其中, $X_{i}^{T}$ 是 $X_{i}$ 的转置矩阵, $\operatorname{trace}(\bullet)$ 表示矩阵的迹。

将两类运动想象 $R_{1}$ 和 $R_{2}$ 相加即可得 $\mathrm{EEG}$ 信号的混合 空间协方差矩阵, 并对和进行谱分解:

$$
R_{c}=R_{1}+R_{2}=U_{c} \lambda_{c} U_{c}^{T}
$$

其中, $U_{c}$ 是 $R_{c}$ 的特征向量矩阵; $\lambda_{c}$ 是 $R_{c}$ 的特征向 量对应的特征值所组成的对角阵。计算白化矩阵 $W$ 如下 式:

$$
W=\lambda_{c}^{(-1 / 2)} U_{c}^{T}
$$

$R_{c}$ 可以进行如下变换:

$$
S_{c}=W R_{c} W^{T}
$$

将矩阵白化之后, $R_{1}$ 和 $R_{2}$ 具有相同的特征值, 具有 如下性质:

$$
\left\{\begin{array}{c}
S_{1}=B \lambda_{1} B^{T} \\
S_{2}=B \lambda_{2} B^{T} \\
\lambda_{1}+\lambda_{2}=I
\end{array}\right.
$$

其中, $B$ 是 $S_{1}$ 和 $S_{2}$ 共同的特征向量, $I$ 是单位矩阵。

两类脑电信号之间的空间滤波器即为 $Q=\left(B^{T} W\right)^{T}$, 将一类 $E E G$ 信号通过空间滤波器后可得 $Z_{i}=Q X_{i}$ 。可得特 征向量 $f_{p}$ :

$$
f_{p}=\log \left(\operatorname{var}\left(Z_{p}\right) / \sum_{i}^{2 m} \operatorname{var}\left(Z_{i}\right)\right)
$$

其中 $Z p(p=1, \ldots 2 m)$ 。本文取 $m=2$, 构造一组四维特 征向量。

\section{2. 支持向量机}

支持向量机(Support Vector Machine, SVM)是一种有 监督的机器学习算法, 主要用于分类和回归分析。最早在 1995年, SVM由 Cortes 和 Vapnik 两人提出, 主要用于
解决小样本问题、非线性问题和高维特征分类问题, 现在 已经成为机器学习算法的研究热点 [13]。支持向量机主要 思想是通过某种事先选择的非线性映射( 核函数) 将线性 不可分的空间映射到一个高维的线性可分的特征空间, 在 这个空间利用结构风险最小化原则构造最优分类超平面， 使分类面两侧相距此平面最近的不同种类样本之间间隔 最大[14], 在对最优超平面的构造问题上可通过在约束条 件

$$
y_{i}\left[w \bullet \Phi\left(x_{i}\right)+b\right] \geq 1-\zeta_{i} ; \zeta_{i} \geq 0,1,2, \ldots, l
$$

下使得函数

$$
\Phi(w)=\frac{1}{2}\|w\|^{2}+C \sum_{i=1}^{l} \zeta_{i}
$$

取得最小值。式中, $x$ 为支持向量机的输入向量, $y_{i}$ 为 $x_{i}$ 所属类别, $w$ 分类面的权系数向量, $b$ 为分类域值, $C>0$ 是惩罚因子, $C$ 越大表示对错误分类的惩罚越大, $\xi_{i}$ 为松弛因子。 $\phi$ 将样本从输入空间映射到特征空间。上 述优化问题可以用下式求解

$$
\begin{gathered}
\underbrace{\max }_{\alpha} W(\alpha)=\sum_{i=1}^{l} \alpha_{i}-\frac{1}{2} \sum_{i=1}^{l} \sum_{j=1}^{l} \alpha_{i} \alpha_{j} y_{i} y_{j} K\left(x_{i}, x_{j}\right), \\
\text { s.t. } y^{T} \alpha=0
\end{gathered}
$$

式 中 , $0 \leq \alpha_{i} \leq C(i=1, \ldots, l), 0 \leq \alpha_{j} \leq C(j=1, \ldots, l), i \neq j$, $K\left(x_{i}, x_{j}\right)$ 为核函数, $\alpha_{i}$ 为拉格朗日乘子, 若 $\alpha_{i}>0$, 则称相应的样本 $x_{i}$ 为支持向量。应用拉格朗日乘 子求解, 得最终的分类函数如下式:

$$
y(x)=\operatorname{sign}\left[\sum_{i=1}^{l} \alpha_{i} y_{i} K\left(x_{i}, x\right)-b\right]
$$

核函数是SVM的重要组成部分, 常用的核函数有线性 核函数、二次核函数、多项式核函数、径向基核函数等等 [15]。本文采用线性核函数。

\section{4. 数据处理及分析}

\section{1. 预处理}

为了使数据尽可能去除其他因素的影响, 且具有代表 性, 首先对原始EEG信号做预处理。已知CSP算法需要大 量的电极和对特定的脑电信号通道分析。根据脑生理结构 和相关文献研究成果, 本文对中央区和顶叶的 25 个电极进 行分析, 包括[16]: Fz F1 F2 FCz Fc1 Fc2 Fc3 Fc4 Fc5 Fc6 Cz C1 C2 C3 C4 C5 C6 CP1 CP2 CP3 CP4 CP5 CP6 Pz POz。 研究表明, 人们在进行单侧手部运动想象时, 其对侧运动 感觉区的 $\mu$ 节律 （8-13 Hz）和 $\beta$ 节律 $(18-30 \mathrm{~Hz})$ 会出现 幅值降低的现象，称为事件相关去同步 (Event-Related Desynchronization, ERD）; 其同侧运动感觉区的 $\mu$ 节律和 $\beta$ 节律出现幅值升高的现象, 称为事件相关同步 
（Event-Related Synchronization, ERS）[17]。因此, 本研 究选择Butterworth 带通滤波器对EEG信号的样本进行 8-30 Hz带通滤波。图3和图4展示了某次样本滤波前和滤 波后 $\mathrm{C} 3$ 和 $\mathrm{C} 4$ 的通道信号, 滤波后的EEG较好地消除了噪 声信号及无关思维信号的影响。

运动想象脑电信号的幅度大概在5微伏左右, 图3和图 4所使用的数据为右手运动想象数据, 可看出在第 1000 个 采样点后, 与右手同侧的 $\mathrm{C} 4$ 通道幅值较大, 对侧的 $\mathrm{C} 3$ 通 道幅值则较小, 体现了一定的ERS/ERD现象。考虑到不同 受试者存在差异性, 因此本研究经过对比后, 选取 $3 \mathrm{~s}$ 数据 中的 $0.5 \mathrm{~s}-2 \mathrm{~s}$ 作为后续分析使用。为了后续数据处理速度运 行快一点, 本研究将采样率降低为 $512 \mathrm{~Hz}$, 此时每人每周 采集的数据大小为 $25 \times 768 \times 120 \times 2$, 其中 25 为通道数, 768 为采样点数, $120 \times 2$ 为动作次数。

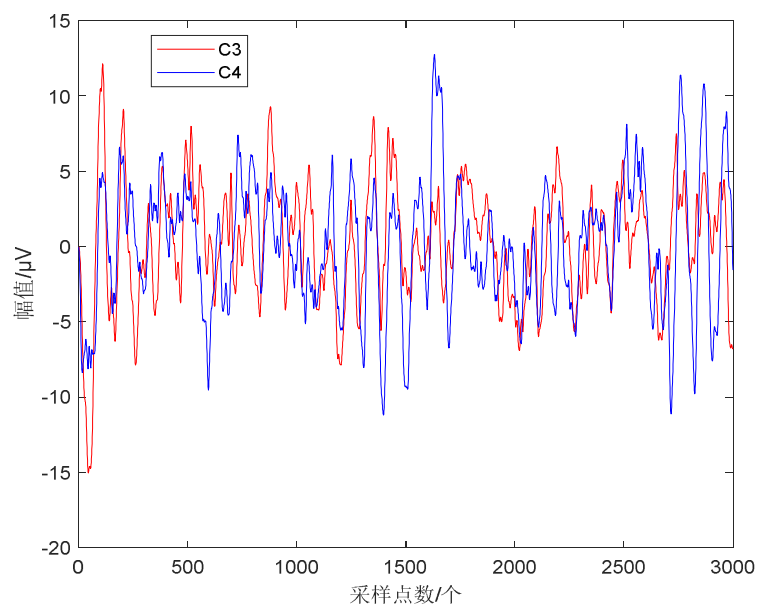

图3 原始 $\mathrm{C} 3$ 和 $\mathrm{C} 4$ 通道的 $\mathrm{EEG}$ 信号。
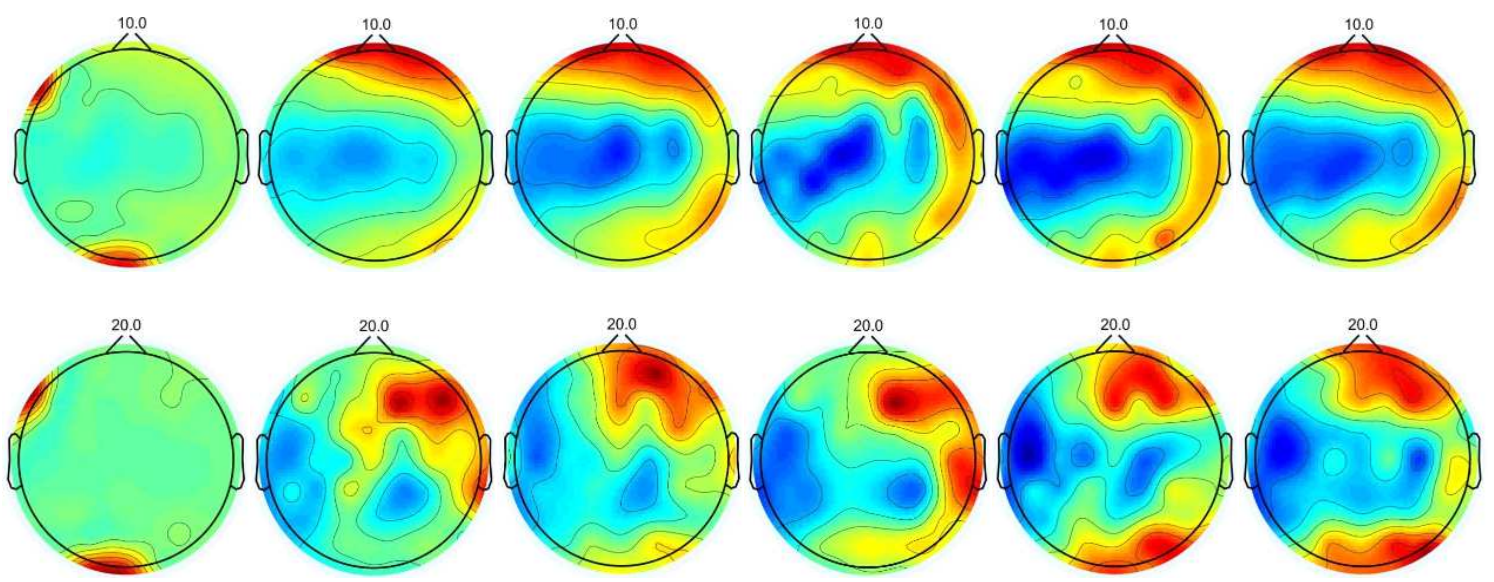

图5 受试者A（残疾人） $\mu$ 节律和 $\beta$ 节律波的脑地形图。
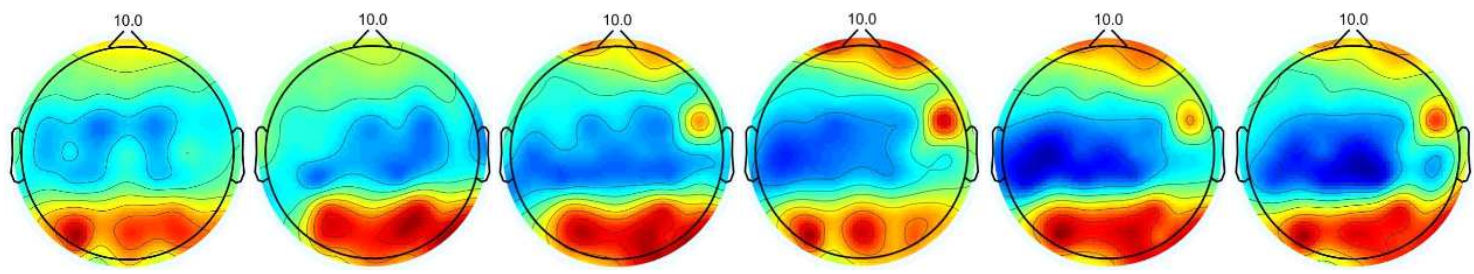

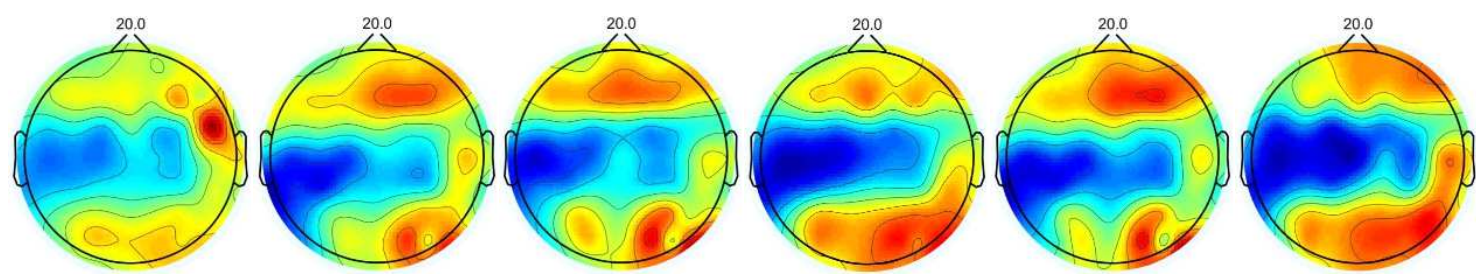

图6 受试者C（正常人） $\mu$ 节律和 $\beta$ 节律波的脑地形图。

从图 5 和图 6 中可以看出, 由于运动想象训练不断地刺 激着大脑运动皮层的神经元, 两名受试者的ERD/ERS现象 都越来越明显。受试者 $\mathrm{A}$ 在训练前执行运动想象任务时, 几乎不存在 $\mu$ 节律波和 $\beta$ 节律波, 而受试者 $\mathrm{C}$ 一开始就可以 激发出较弱的 $\mu$ 节律波和 $\beta$ 节律波。对比发现, 五周训练过 程中, 受试者 $\mathrm{A}$ 的大脑能量分布图越来越接近正常人, ERD/ERS现象出现的区域也明显集中在大脑的运动区, 并 且运动区能量值与非运动区能量值的差异也越来越明显。 对比其他两名受试者同样可以都得到以上结果, 这里就不 作图了。

\section{3. 特征分类}

本文还采用共同空间模式和支持向量机对EEG信号 进行特征提取及分类。先通过左右手脑电信号计算得到平 均归一化空间协方差矩阵, 然后计算获得混合空间协方差 矩阵, 再求得投影矩阵 $\mathrm{W}$; 对 120 组运动想象脑电数据进 行处理, 计算求得特征向量矩阵, 脑电特征为特征向量矩
阵各行的方差。本文选择了 25 个通道, $\mathrm{m}=2$, 则单个动作 的特征数为 4 。本文采用随机的方法对每周的数据区分训 练集和测试集, 其中训练集和测试集的数量比为 $3: 1$ (每 人每周采集 $120 \times 2$ 个动作）, 四名受试者训练五周的所有 数据分类结果见表 1 。根据表 1 中的数据, 本研究还将每名 受试者训练结果绘制成曲线如图7, 其中受试者 $\mathrm{A}$ 和 $\mathrm{B}$ 是失 去左手的残疾人, $\mathrm{C}$ 和 $\mathrm{D}$ 是正常人。

表1 每名受试者训练前后两类动作分类正确率。

\begin{tabular}{lllll}
\hline \multirow{2}{*}{ 训练时间 } & 受试者 & & & \\
\cline { 2 - 5 } & $\mathbf{A}$ & $\mathbf{B}$ & $\mathbf{C}$ & $\mathbf{D}$ \\
\hline 训练前 & $69.38 \pm 3.36$ & $73.76 \pm 1.49$ & $74.04 \pm 1.68$ & $70.35 \pm 4.30$ \\
训练一周 & $74.05 \pm 1.68$ & $79.30 \pm 2.78$ & $79.95 \pm 2.20$ & $79.85 \pm 1.45$ \\
训练两周 & $81.32 \pm 1.56$ & $82.78 \pm 2.38$ & $84.65 \pm 1.04$ & $86.20 \pm 2.47$ \\
训练三周 & $83.97 \pm 1.21$ & $84.93 \pm 1.74$ & $86.96 \pm 1.72$ & $90.33 \pm 1.98$ \\
训练四周 & $85.53 \pm 1.69$ & $85.65 \pm 1.41$ & $91.22 \pm 2.27$ & $93.13 \pm 2.07$ \\
训练五周 & $85.64 \pm 1.41$ & $86.96 \pm 1.72$ & $91.34 \pm 1.92$ & $93.84 \pm 2.18$ \\
\hline
\end{tabular}

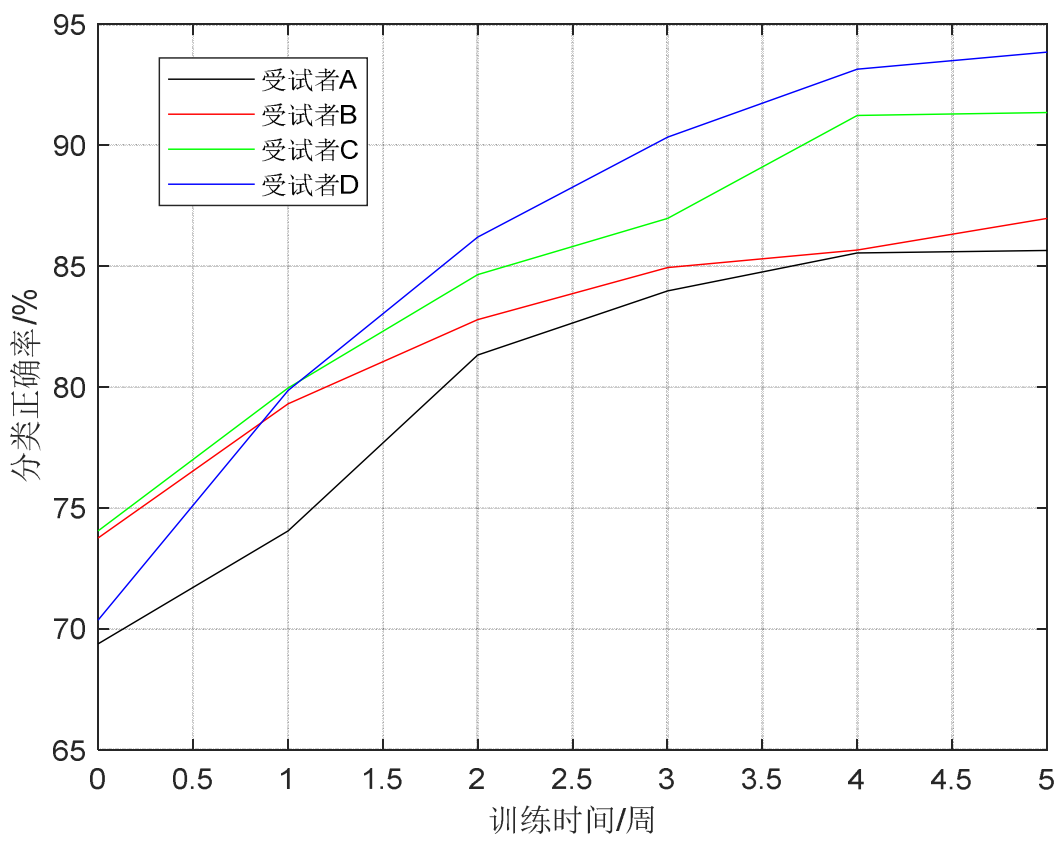

图7 训练结果曲线图。

从表 1 和图 7 , 我们可以看到经过五周的运动想象训练, 左右手动作分类正确率都有明显的提高, 其中残疾人可以 达到 $85 \%$ 以上, 正常人可以达到 $90 \%$ 以上。从图 7 中可以看 出, 残疾人的分类正确率在第三周到第四周增长比较缓慢, 正常人呈现出比较明显的增长趋势; 在第四周到第五周, 残疾人和正常人均出现了缓慢变化的趋势。
将脑地形图的结果和分类结果结合分析, 发现分类正 确率的提高和ERD现象明显程度基本相符合, 两者都说明 了训练对大脑可塑性的影响, 不断的训练过程就是大脑运 动区域的一个学习和记忆的过程。由于残疾人大脑的运动 功能已经收到了损坏, 总体训练结果会比正常人稍微不理 
想一些, 但是总体来说结果训练对大脑运动功能是有恢复 作用的。

\section{5. 总结与展望}

本文通过设计残疾人和正常人的对照实验来研究训 练对人大脑可塑性的影响, 实验中对四名受试者进行了五 周的运动想象训练。根据采集的脑电信号, 本文从脑地形 图的能量分布图分析了训练过程中的ERD/ERS现象的变 化情况, 并采用 CSP $+\mathrm{SVM}$ 的改进算法对左右手两类动作 进行分类, 得到了残疾人分类正确率 $85 \%$ 以上和正常人分 类正确率 $90 \%$ 以上的结果。实验表明, 运动想象训练可以 帮助残疾人恢复大脑与运动相关的区域的某些功能, 也可 以加强大脑激发出的 $\mu$ 节律波和 $\beta$ 节律的强度。并且, 本文 通过左右手动作分类说明通过训练可以增加左右手脑电 信号的差别, 从而提高不同动作的分类正确率。本研究的 成果充分说明了训练对大脑的可塑性, 也为残疾人恢复运 动功能提供了科学的依据。本人今后将继续研究运动想象 训练的多分类问题。

\section{致谢}

在选题和研究过程中, 本课题得到了尹老师的亲切关 怀和悉心指导。她严谨的科学态度和严谨的学术精神深深 地感染和激励着我。同时, 我也要感谢参与实验的四位志 愿者以及和我一起收集脑电图信号的学长们。最后, 我也 要感谢本论文所引用的各位学者的专著, 如果没有这些学 者的研究成果的启发和帮助, 我将很难完成本篇论文的最 终写作。

\section{参考文献}

[1] 李敏, 徐光华, 谢俊, 等. 脑卒中意念控制的主被动运动康 复技术 $[J]$. 机器人, 2017 (5): 759-768.

[2] Wolpaw J R, Birbaumer N, McFarland D J, Pfurtscheller G, Vaughan T M. Brain-Computer Interfaces for Communication and Control, Clin. Neurophysiol., 2002; 113: 767-791.

[3] 张瑞芬. 基于 CCA的ICA算法及其在EEG信号处理中的应 用研究[D].西安电子科技大学, 2017 .

[4] Tang $\mathrm{Z}, \mathrm{Li} \mathrm{C}, \mathrm{Wu} \mathrm{J}$, et al. Classification of EEG-based single-trial motor imagery tasks using a B-CSP method for BCI [J]. Frontiers of Information Technology \& Electronic Engineering, 2019, 20 (8): 1087-1098.
[5] COSTAGLiOLA S, SENO B D, MATTEUCCI M. Recognition and classification of P300s in EEG signals by means of feature extraction using wavelet decomposition [C]. Proceedings of International Joint Conference on Neural Networks. Atlanta, USA, 2009:597603.

[6] 王行愚, 金晶, 张宇, 等. 脑控:基于脑 - 机接口的人 机融合控制 $[\mathrm{J}]$. 自动化学报, 2013，39(3):208-221.

[7] 高上凯. 浅谈脑 - 机接口的发展现状与挑战 [J]. 中 国生物医学工程学报, 2007，26(6):801-809.

[8] 程明. 基于脑电信号的脑 - 计算机接口的研究 [D]. 北 京:清华大学, 2004.

[9] 刘冲, 赵海滨, 李春胜. 基于 CSP 与 SVM 算法的运动 想象脑电信 号分类 $[\mathrm{J}]$. 东北大学学报, 2010, 31(8):1098 -1101 .

[10] Ramoser H, Muller-Gerking J, Pfurtscheller G. Optimal spatial filtering of single trial EEG during imagined hand movement $[\mathrm{J}]$. IEEE Transactions on Rehabilitation Engineering, 2000, 8 (4): 441-446.

[11] 王娜. 基于脑电信号的运动想象分类研究[D]. 南京邮电大 学,2018.

[12] 赵凯. 多类运动想象脑电信号识别及其在BCI中的应用研究 [D].东北电力大学, 2019 .

[13] Vapnik V N. Statistical learning theory [M]. New York: Jone Wiley\&Sons, 1998.

[14] 庄玮,段锁林,徐亭婷. 基于SVM的4类运动想象的脑电信号 分类方法 [J].常州大学学报(自然科学版),2014,26(01):42-46.

[15] 杨淑莹. 模式识别与智能计算--Matlab技术实现 $[M]$. 北京: 电子工业出版社, 2008.

[16] 王毅军. 结合能量和相位特征的多分类想象运动脑-机接口 [C]. 中国电子学会生物医学电子学分会、中国生物医学工 程学会生物医学测量分会、中国生物医学工程学会生物信 息与控制分会、中国生物医学工程学会生物医学传感器技 术分会. 中国生物医学工程进展—2007中国生物医学工 程联合学术年会论文集 (下册).中国电子学会生物医学电 子学分会、中国生物医学工程学会生物医学测量分会、中 国生物医学工程学会生物信息与控制分会、中国生物医学 工程学会生物医学传感器技术分会: 中国生物医学工程学 会,2007:691-694.

[17] ROSSO OA, MARTIN MT, FIGLIOLAA, et al. EEG analysis using wavelet-based information tools [J]. J Neurosci Methods, 2006, 153 (2): 163-182. 